\title{
A CASE OF PARTIAL ANODONTIA
}

\author{
Captain G. D. STAFFORD, L.D.S., R.A.D.C. (T.A.) \\ Dental Officer, 7th (Manchester) General Hospital, R.A.M.C. (T.A.) *
}

Introduction and History

This patient was referred to the Department of Maxillo-Facial and Oral Surgery, Withington Hospital, Manchester, on his voluntary discharge from the Army. He presented as a case of partial anodontia with gross overclosure of the vertical dimension of the lower facial height.

The patient was born in December 1943, and had lived with his defect during the last few years without any particular handicap, but he was now concerned with the loss of aesthetics and loss of function. He presented with no relevant general medical history; and the only past dental history was that $21 / 22$ had been extracted more recently "because they ached".

There was no family history of anodontia although a father who had been edentulous for many years might be considered a possibility.

On examination the appearance was that of an edentate with a slightly pronounced chin pad. He had a normal physique and appeared in good physical condition. The skin texture was good and hair and nails normal. In a position of rest the patient looked normal (Figure 1), but when he attempted to occlude his teeth the gross overclosure became evident (Figure 2).

On intra-oral examination the following teeth were noted present-

$$
\frac{7 \text { edc } / \cdot \operatorname{cd} 78}{7 \text { edcba } / \operatorname{abcde} 7}
$$

L was submerged. Figure 3 shows a maxillary and madibular model mounted in such a way as to demonstrate the separation of the teeth at rest. His freeway space was $15 \mathrm{~mm}$.

Radiographs showed the presence of an unerupted $8 \%$, and that the roots of the dediduous teeth were resorbed. $6 / 6$ and $\overline{6 / 6}$ had been extracted in childhood.

\section{Treatment}

It was decided that the remaining teeth should be retained, and that overlay dentures should be constructed. $\overline{7 / 7}$ showed early caries in the fissures on the occlusal surfaces, and these were eliminated and amalgam restorations placed in these teeth.

Impressions were then taken of the teeth, and an overlay occlusal rim constructed so that the centric relationship could be obtained. The models were then mounted on an anatomical articulator. It was found on surveying of the natural teeth that undercuts existed only mesio-lingually on $\overline{7 / 7}$ which were slightly tilted in that direction. It was decided therefore to construct an all gold cast splint that would fit over the existing teeth, and an occlusal form was developed on the surface of this splint so that retention and support could be gained for an overlay denture. Gold was chosen as the material for the splint so that it might be adapted around the cervical areas more easily at insertion. Gold was also used for the metal elements of the overlay denture.

*Present address: Department of Prosthetics, Turner Dental School, University of Manchester, Manchester 15. 


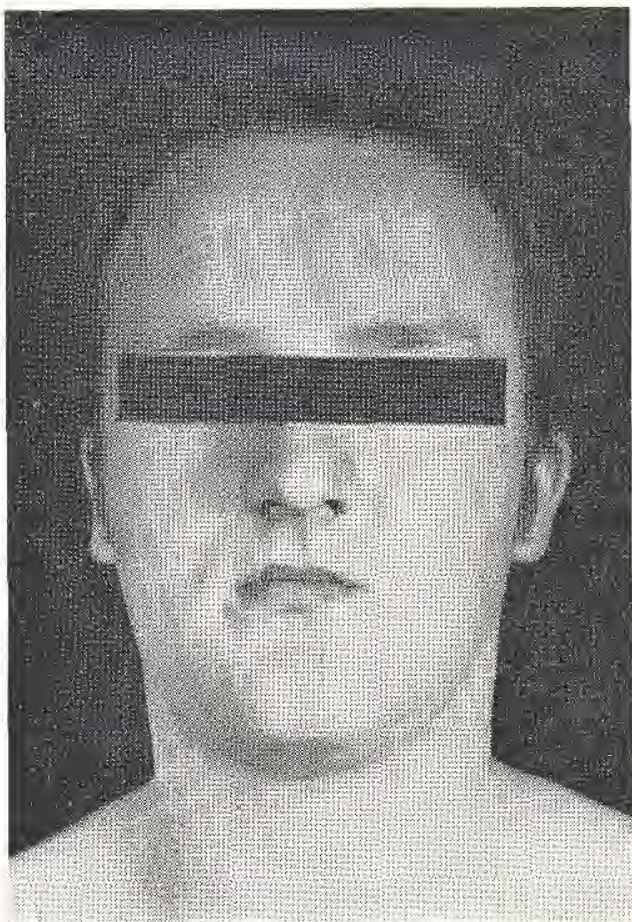

Fig. 1

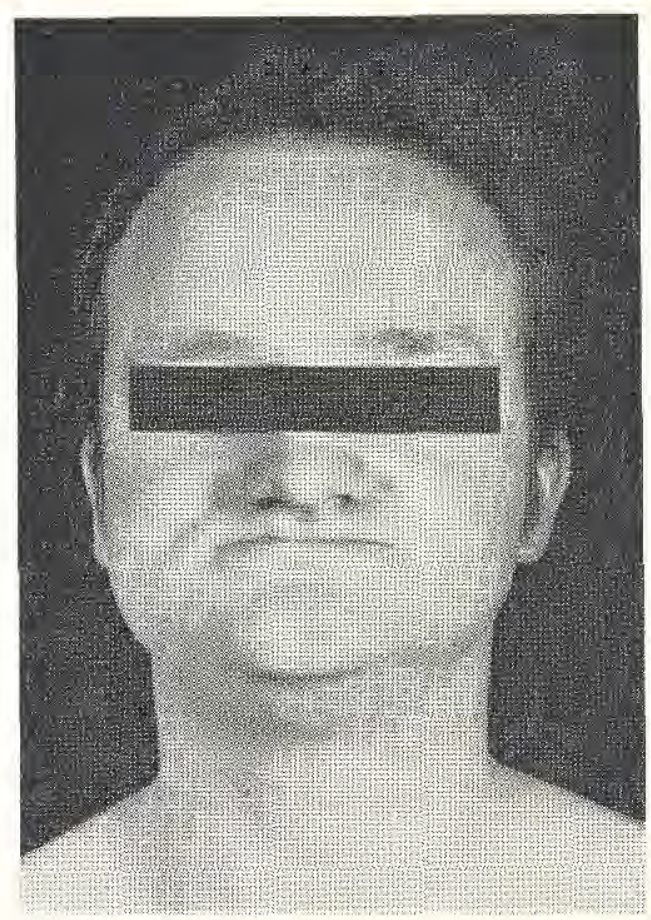

Fig. 2 
Special care was taken with the final impression so that a good reproduction of the cervical area of the teeth might be produced. Zinc chloride cotton wisps were placed around the gingival margins, and after their removal an alginate impression was taken immediately, and a detailed impression obtained of the gingival area of the teeth.

The splints were made in such a way that the 'skirt' around each tooth was carried subgingivally wherever possible. After casting, the splints were tried in place and Figure 4 shows these on the models. The splints were cemented in place with black copper cement. The artificial teeth were then set up on the gold skeleton which had retainers engaging undercuts on the gold splint and after trying the waxed-up trial dentures in the mouth, and making any necessary adjustments, the dentures were processed in polymethylmethacrylate. Figure 5 shows the dentures with the incorporated gold skeleton. Figures 6 and 7 show the dentures in the mouth.

The dentures have now been worn for twelve months, and the patient is delighted with the function and aesthetics.

\section{Discussion}

The treatment plan for the procedure just described was decided upon after consideration of the following points.

1. Loss of the deciduous teeth would leave the patient with $7 / 78$ and $\overline{7 / 7}$, and these would be inadequate support for partial dentures of the size required to restore aesthetics and function.

2. Loss of all the teeth would make large dentures necessary on account of the large freeway space, and the use of a hollow-box or granules to reduce the weight would still mean that the dentures would be large.

3. Lack of development of the permanent teeth, particularly in the anterior mandibular region had led to no alveolar development, and if the patient was rendered edentulous now, then the prognosis for any adequate alveolar support for full dentures in the years ahead was bad.

4. Lack of undercuts upon the teeth make the development of retention for a normal overlay denture difficult.

5. Lack of bone support for the deciduous teeth means that any appliance that was being repeatedly inserted and removed by the patient would loosen the teeth, and they would soon be lost. A splint would help to retain the deciduous teeth by spreading the load.

6. If nothing was done then deciduous teeth would soon be lost by the forces of chewing and the patient's problem of lack of aesthetics would not be solved.

7. There are inadequate abutments for any fixed prosthesis of the conventional type.

It was realised when treatment was undertaken that the life of the remaining natural teeth was very limited, and it was felt that any procedure that would prolong the life of the deciduous teeth, and help the patient through even only a few years, would be worthwhile.

The biggest problem associated with this particular case was the production of an adequate margin to the gold splint so as to avoid periodontal breakdown. As explained above, considerable care was taken in the impression technique to obtain the best posible reproduction of the cervical areas of the teeth so as to enable the technician to finish the casting as subgingival as possible. Where there were spaces between the teeth the 


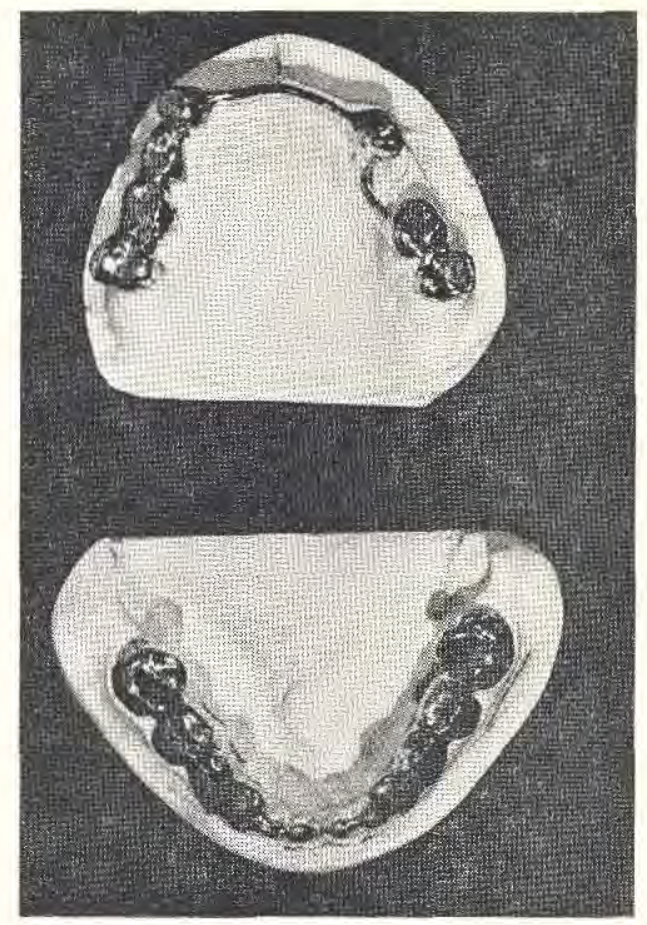

Fig. 4

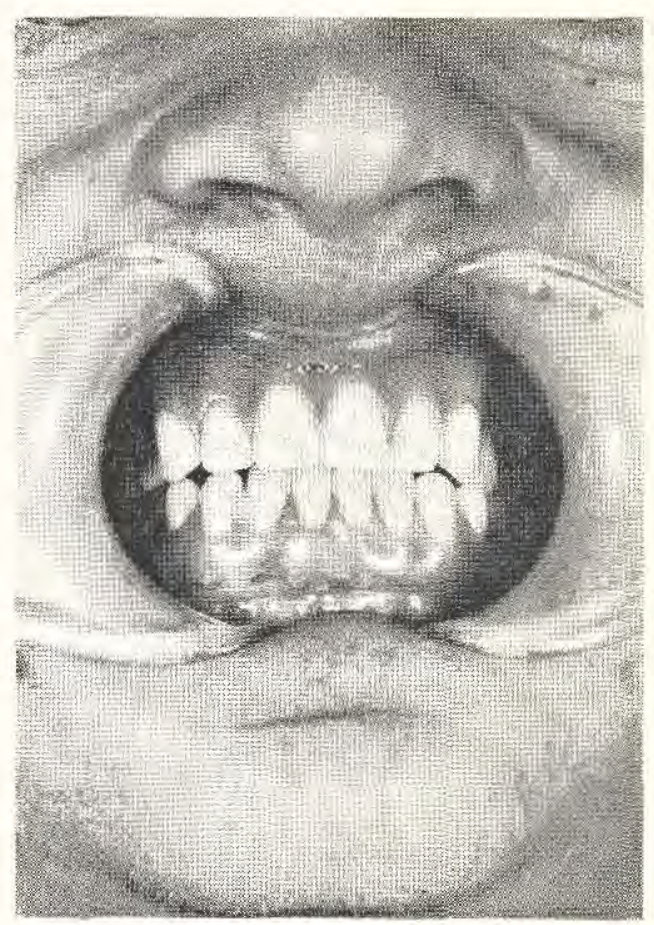

Fig. 6

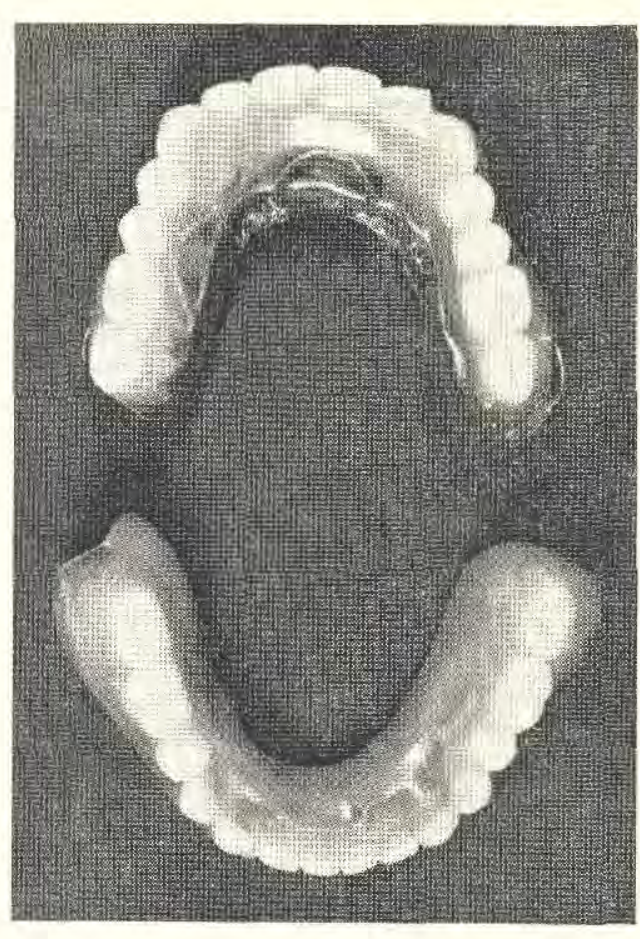

Fig. 5

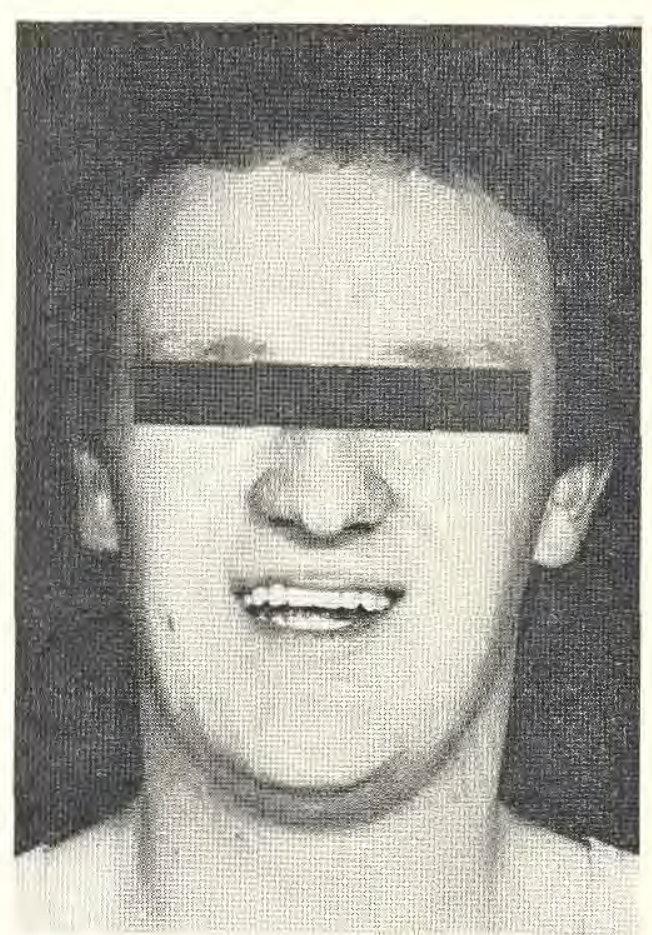

Fig. 7 
casting was so arranged that softwood sticks could by used to clean interdentally. So far the periodontal condition of the patient has remained good.

It does seem reasonable, upon reflection, that it might have been wise to have extracted $\overline{b a / a b}$ and to have placed a bar of the Dolder type between $\overline{c / c}$ on the splint.

\section{Conclusion}

An unusual case of partial adodontia, first recognised at the recruit dental examination, is treated with the insertion of overlay dentures upon cemented splints.

\section{Acknowledgements}

I am greatly indebted to Mr. A. Weldon Moule, F.D.S.R.C.S., Senior Consultant Oral Surgeon and Head of Department of Maxillofacial and Oral Surgery at Withington Hospital, Manchester for permission to treat and publish this case. I also wish to thank Brigadier D. V. Taylor, C.B.E., H.D.D., F.D.S.R.C.S., Consulting Dental Surgeon to the Army for referring this case to this Unit. My thanks are also due to Mr. Byrne, of the Regional Dental Laboratory, Withington, whose skilful and willing technical assistance contributed greatly to the success of this case, 